\title{
Giant current-driven domain wall mobility in (Ga,Mn)As
}

\author{
Anh Kiet Nguyen, Hans Joakim Skadsem, and Arne Brataas \\ Department of Physics, Norwegian University of Science and Technology, N-7491, Trondheim, Norway
}

\begin{abstract}
We study theoretically hole current-driven domain wall dynamics in (Ga,Mn)As. We show that the spin-orbit coupling causes significant hole reflection at the domain wall, even in the adiabatic limit when the wall is much thicker than the Fermi wavelength, resulting in spin accumulation and mistracking between current-carrying spins and the domain wall magnetization. This increases the out-of-plane non-adiabatic spin transfer torque and consequently the current-driven domain wall mobility by three to four orders of magnitude. Trends and magnitude of the calculated domain wall current mobilities agree with experimental findings.
\end{abstract}

Spin-polarized currents can reverse the magnetization, excite spin-waves or move domain walls in ferromagnets. These are intriguing phenomena which can become useful for magnetic memories. Ferromagnetic semiconductors are especially interesting because the critical current density $\left(j_{c}\right)$ required to move domain walls is two to three orders of magnitude smaller than in ferromagnetic metals [1, 2, 3, ,4]. Much effort has been invested experimentally $[1,2,3,4,5,5,6]$ and theoretically $7,8,19,10,11,12,13,14,15]$ on current-driven domain wall dynamics [16]. However, it is not understood why the critical current density for domain wall motion is so small in semiconductors compared to metals.

The spin-current induced torque on domain walls can be written as a sum of an in-plane part and an out-ofplane part, $\boldsymbol{\tau}=\boldsymbol{\tau}^{i n}+\boldsymbol{\tau}^{\perp}$. The in-plane (out-of-plane) torque is spanned by (perpendicular to) the gradient of the local magnetization. In ferromagnetic metals, the domain wall width $\left(\lambda_{w}\right)$ is large compared to the Fermi wavelength $\left(\lambda_{F}\right)$ and the spin-orbit coupling is weak. Here, the domain wall does not reflect electrons which adiabatically align their spin close to the local magnetization direction as they traverse the domain wall. The associated angular momentum transfer induces an in-plane torque on the domain wall [8, 9, 10, 11, 12, 13, 14]

$$
\boldsymbol{\tau}^{i n}=-j_{s} \mathbf{m} \times[\mathbf{m} \times(\hat{\jmath} \cdot \nabla) \mathbf{m}] .
$$

$\tau^{i n}$ is also called the adiabatic spin torque. In Eq.(1), $j_{s}$ is the spin current density. The unit vectors $\hat{\jmath}$ and $\mathbf{m}$ are in the directions of the current and the local magnetization, respectively. At low current densities, $\tau^{i n}$ does not cause any steady-state motion of the domain wall [10, 11]. However, even a small out-of-plane torque $\tau^{\perp}$ can induce a finite domain wall drift velocity [12, 13, 14]. An often used approximate form for the out-of-plane torque is 12,13 ]

$$
\boldsymbol{\tau}^{\perp}=-\beta j_{s} \mathbf{m} \times(\hat{\jmath} \cdot \nabla) \mathbf{m},
$$

where $\beta$ is a small dimensionless parameter. The domain wall drift velocity is controlled by the out-of-plane torque and is proportional to $\beta[12,13]$. In literature, deviations from the adiabatic in-plane torque, e.g. $\tau^{\perp}$, are often called the non-adiabatic torque. We prefer to classify the torques as in-plane and out-of-plane, where the latter can induce a steady state domain wall motion.

In this Letter, we show that the intrinsic spin-orbit coupling in the valence band of magnetic III-V semiconductors strongly enhances the current-induced outof-plane torque and thereby the domain wall velocity. $\tau^{\perp}$ depends non-locally on the whole domain wall profile and cannot be described by the local expression given by Eq.(2). We find that the steady-state domain wall velocity $\left(v_{w}\right)$ is proportional to the current density $(j)$. The relevant measure of current-driven domain wall motion is the domain wall current-mobility $\mu_{I}=v_{w} / j$. Using realistic values for domain wall width and spin-orbit coupling, we find that $\mu_{I}$ is enhanced by three to four orders of magnitude compared to a system with weak or vanishing spin-orbit coupling. This may explain an open question: why $\mu_{I}$ (or $j_{c}$ ) is much larger (smaller) in $(\mathrm{Ga}, \mathrm{Mn})$ As than in ferromagnetic metals [1, 2, 3, 4].

In conventional ferromagnetic metals only a very small fraction of the electrons contribute to the out-of-plane torque via spin-flip scattering at extrinsic impurities or via non-adiabatic corrections due to a finite domain wall width [12, 13, 14]. Radically different from this, the strong intrinsic hole spin-orbit coupling in $(\mathrm{Ga}, \mathrm{Mn}) \mathrm{As}$ causes a finite domain wall resistance even in the adiabatic limit $\left(\lambda_{w} \gg \lambda_{F}\right)$, by preventing a large fraction of holes to adiabatically adjust their spins to the magnetization of the domain wall [18, 19]. The intrinsic spinorbit coupling in combination with the magnetization cause an anisotropic momentum distribution of propagating modes. Fig [1 a), b) and c) show distributions of transverse propagating modes in $(\mathrm{Ga}, \mathrm{Mn}) \mathrm{As}$ for different magnetization directions [18]. Only transverse modes that exist for all magnetization directions within the domain wall, Fig@1), will conduct. The remaining modes are totally reflected [18, 19]. The fraction of reflected holes is large and comparable to the total number of conducting particles. This induces an enhanced domain wall resistance, as well as spin accumulation and mistracking between hole spins and the magnetization of the domain wall. We find that the total out-of-plane torque increases by three to four orders of magnitude compared to systems with vanishing spin-orbit coupling. The current-induced 
mobility is subsequently enhanced approximately proportional to the total out-of-plane torque.

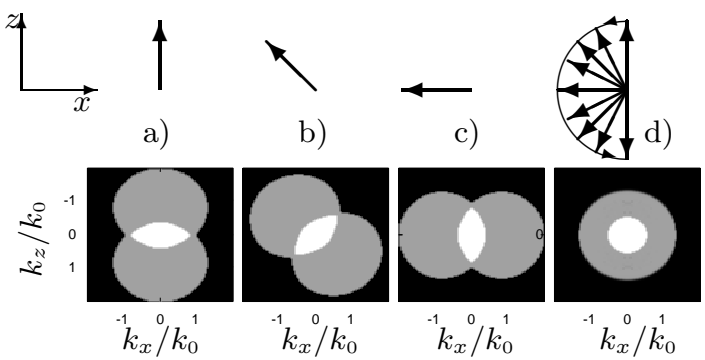

FIG. 1: a), b) and c) Distributions of transverse propagating modes in $(\mathrm{Ga}, \mathrm{Mn})$ As for different magnetization directions shown by the arrows. White (gray) color indicates that two (one) spin channels are open for transport. d) Distribution of transverse conducting channels through an adiabatic Bloch domain wall in $(\mathrm{Ga}, \mathrm{Mn})$ As. Arrows show the magnetization directions itinerant holes encounter while traversing a Bloch wall. For an adiabatic domain wall, transverse modes in a) that do not exist in d) are total reflected by the wall. Such reflections increase the out-of-plane torque on the domain wall which subsequently increases the domain wall drift velocity. The plots are created using Eq.(3) with $\gamma_{1}=6.8, \gamma_{2}=2.7$, $h_{e x} / \epsilon_{0}=1.5, \epsilon_{F} / \epsilon_{0}=2.25$.

We shall use the simplest model that captures the essential physics of holes in (Ga,Mn)As, namely the 4-band Kohn-Luttinger Hamiltonian in the spherical approximation [18, 20, 21, 22]

$$
H=\frac{\hbar^{2}}{2 m}\left[\left(\gamma_{1}+\frac{5}{2} \gamma_{2}\right) p^{2}-2 \gamma_{2}(\mathbf{p} \cdot \mathbf{S})^{2}\right]-\mathbf{h}(\mathbf{r}) \cdot \mathbf{S},
$$

where, $m$ and $\mathbf{p}$ are the bare electron mass and the hole momentum operator, respectively. $\mathbf{S}$ is a vector of $4 \times 4$ dimensionless angular momentum operators for a $S=3 / 2$ spin, and $\gamma_{1}$ and $\gamma_{2}$ are Luttinger parameters. In Eq.(3), $\mathbf{h}$ represents the exchange field from the localized magnetic moments. The exchange field is assumed to have a constant modulus and to be homogeneous in the transverse directions, $|\mathbf{h}(y)|=h_{e x}$. The parameter $\gamma_{2}$ determines the strength of the effective spin-orbit coupling of the holes. For a given doping density, Eq.(3) with $\mathbf{h}=0$ describes hole doped GaAs and provides a Fermi energy $\epsilon_{0}$, a Fermi wavevector $k_{0}=\sqrt{2 m_{0} \epsilon_{0}} / \hbar$ and a Fermi wave length $\lambda_{0}=2 \pi / k_{0}$ for heavy holes. Here, $m_{0}=m / 2.6$ represents the heavy hole mass in GaAs.

Focusing on low temperatures and low bias transport properties, we consider the linear response at a Fermi energy $\epsilon_{F}$. Our system is a discrete, rectangular conductor of lengths $L_{x}, L_{y}, L_{z}$ and lattice constants $a_{x}, a_{y}, a_{z}$ sandwiched between two reservoirs. Born-von Karman boundary condi-tions are assumed in the transverse directions $x$ and $z$.

We model the dynamics of domain walls by the dimensionless Landau-Lifshitz-Gilbert (LLG) equation

$$
\frac{d \mathbf{m}}{d \tilde{t}}=-\mathbf{m} \times \tilde{\mathbf{h}}^{e f f}+\alpha \mathbf{m} \times \frac{d \mathbf{m}}{d \tilde{t}},
$$

where $\mathbf{m}(y)$ should be understood as a unit macrospin for the transverse slice located at $y$, and $\alpha$ is the Gilbert damping constant. The dimensionless time is $\tilde{t}=t / t_{0}$ where $t_{0}=M_{s}\left(\lambda_{0} / 2 \pi\right)^{2} / 2 \gamma A$. Here, $M_{s}, A$ and $\gamma=e / m c$ are the saturation magnetization, spin stiffness and the gyromagnetic ratio, respectively. The effective magnetic field reads

$$
\tilde{\mathbf{h}}^{e f f}=\tilde{\nabla}^{2} \mathbf{m}+\tilde{\mathbf{h}}^{a n} \cdot \mathbf{m}-h_{p d} \boldsymbol{\chi}_{S},
$$

where $\tilde{\nabla}=\left(\lambda_{0} / 2 \pi\right) \nabla$. $\tilde{\mathbf{h}}^{\text {an }}$ is the dimensionless anisotropy field, which is used to control the type and the width of the domain wall. Demagnetization fields for simple geometries can be included in $\tilde{\mathbf{h}}^{a n}$. The last term in Eq.(5) is the contribution to the spin-transfer torque from the non-equilibrium itinerant holes [15, 17]. The dimensionless coupling constant $h_{p d}$ and the spin-density response function $\chi_{S}$ are defined below. The LLG equation is numerically integrated using a fourth-order Runge-Kutta method 24].

We calculate the current carrying wave function by a stable transfer matrix formalism [18, 23]. The nonequilibrium spin density $\langle\rho \mathbf{S}\rangle^{n e}$ is determined as a trace over all hole states between $\epsilon_{F}$ and $\epsilon_{F}+e V$ in the left reservoir. We define the linear response function for the spin density as $\boldsymbol{\chi}_{S}=\left(e \hbar k_{0} / j m_{0}\right)\langle\rho \mathbf{S}\rangle^{n e}$, which gives $h_{p d}=\hbar h_{e x} \lambda_{0} j / 8 \pi e A \epsilon_{0}$, that is proportional to the current density $j$. Similarly, we also compute the non-equilibrium spin current density, $\mathbf{j}_{s}$, and the average spin per conducting holes, $\langle\mathbf{S}(y)\rangle=\mathbf{j}_{s} e / j \hbar$.

The parameters describing our conductor are $a_{x} / \lambda_{0}=$ $a_{z} / \lambda_{0}=0.2 / 2 \pi, a_{y} / \lambda_{0}=0.75 / 2 \pi, L_{x}=L_{z}=51 a_{x}$ and $L_{y}=400 a_{y}$. Furthermore, $h_{e x} / \epsilon_{0}=1.5$ and $\epsilon_{F} / \epsilon_{0}=2.25$. The ratio $\epsilon_{F} / h_{e x}=1.5$ is in the same order as experimental values 22]. The anisotropy/demagnetization field $\tilde{h}_{x}^{a n}=0$, $\tilde{h}_{y}^{a n}=-1$ for simplicity and to ensure that the considered domain walls are Bloch walls, consistent with recent experiments [1, 2, 5]. We use $\tilde{h}_{z}^{a n}$ to control the domain wall width $\lambda_{w} / \lambda_{0}=\sqrt{1 / \tilde{h}_{z}^{a n}} / 2 \pi$. The Gilbert damping constant is assumed to be $\alpha=0.03$ [25].

The transfer of momentum and angular momentum from holes to the domain wall must be treated on an equal footing. We achieve this as follows: First, we find the equilibrium $(j=0)$ domain wall configuration by integrating Eq.(4) with $h_{p d}=0$ to a sufficiently large time. The resulting equilibrium domain wall configuration is $m_{x}(y)=1 / \cosh \left(y / \lambda_{w}\right), m_{y}(y)=0$ and $m_{z}(y)=\tanh \left(y / \lambda_{w}\right)$, as expected from an analytical equilibrium solution of Eq.(4). Next, a constant current is applied by choosing a finite $h_{p d}$, and the following two steps are iterated: 1) compute $\boldsymbol{\chi}_{S}$ for a given $\mathbf{h}=h_{e x} \mathbf{m}$ using Eq.(3), and 2) integrate $\mathbf{m}=\mathbf{h} / h_{e x}$ a time $\Delta \tilde{t}$ using Eq.(4). We use $\Delta \tilde{t}=0.2$, which is sufficient for convergence.

Let us first consider a system without spin-orbit coupling, $\gamma_{2}=0$. We consider a $\lambda_{w} / \lambda_{0}=2$ domain wall, close to the experimental values [1, 2]. Numerical calculation 

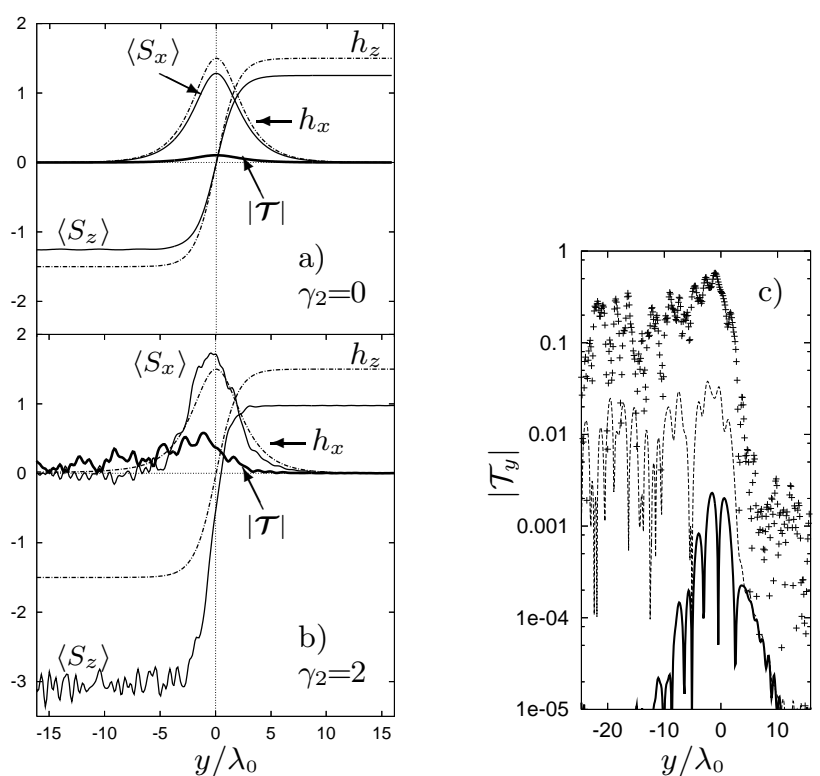

FIG. 2: Steady-state snapshots. a) and b): exchange field $\left(h_{x} / \epsilon_{0}\right.$ and $\left.h_{z} / \epsilon_{0}\right)$, spin per conducting hole $\left(\left\langle S_{x}\right\rangle\right.$ and $\left.\left\langle S_{z}\right\rangle\right)$ and absolute values of spin-torque per conducting hole $|\mathcal{T}|$ as functions of position, for a) vanishing and b) finite spin-orbit coupling. c): absolute values of the out-of-plane torque $\left|\mathcal{T}_{y}\right|$ versus position for $\gamma_{2}=0$ (thick solid line), $\gamma_{2}=0.5$ (dashed line) and $\gamma=2($ symbol +$)$. For all plots, $h_{p d}=0.001$ and $\lambda_{w} / \lambda_{0}=2$.

of the conductance shows that only $0.1 \%$ of the incoming holes are reflected back by the domain wall. From Fig 2 2 ), we see that the hole spins follow the domain wall magnetization closely, and $\langle\mathbf{S}(y)\rangle$ is virtually parallel to $\mathbf{h}(y)$ throughout the system. Outside the wall, $\left\langle S_{z}\right\rangle \approx \pm 1.25$, since both the heavy $\left(S_{z} \approx-1.5\right)$ and light $\left(S_{z} \approx-0.5\right)$ holes participate in the transport. The absolute values of the dimensionless spin-torque per conducting holes, $\mathcal{T}=\left(\mathbf{h} / \epsilon_{0}\right) \times \boldsymbol{\chi}_{S}$, is shown in Fig, 2h). It agrees well with the in-plane torque given by Eq.(1) through the relation $\mathcal{T}=\boldsymbol{\tau}^{i n} e \lambda_{0} / 2 \pi j \hbar$. The out-of-plane torque is very small, as expected for this case.

As shown in Fig 2b), turning on the spin-orbit coupling completely changes the physical picture. Numerical calculation of the conductance shows that the domain wall reflects $45 \%$ of the holes, causing spin accumulation and mistracking between carrier spins and the magnetization of the domain wall, particularly on the upstream side of the wall. Interference between incoming and reflected holes creates a spin-wave pattern in $\langle\mathbf{S}\rangle$ and $\mathcal{T}$, causing the reproducible "noise" in the figures. The shape of the domain wall is, however, virtually unchanged, due to the small current applied, $h_{p d}=0.001$. From our previous study, we know that the majority of reflected holes consists of heavy holes [18, in agreement with $\left\langle S_{z}\right\rangle \approx-3.0$ on the left side of the domain wall.

As discussed in the introduction, the steady state domain wall velocity is controlled by the out-of-plane torque
12, 13. Fig 22) shows the absolute value of the out-ofplane torque component, $\left|\mathcal{T}_{y}\right|$, for three different spinorbit couplings. The spatial total of $\left|\mathcal{T}_{y}\right|$ denoted as $\left\langle\left|\mathcal{T}_{y}\right|\right\rangle=\int d y\left|\mathcal{T}_{y}\right|$ may be used as an estimate of the outof-plane torque contributions to the domain wall drift velocity. Note that even when the spin-orbit coupling vanishes, the out-of-plane torque is finite since the system is not entirely adiabatic, $\lambda_{w} / \lambda_{0}=2 \ll \infty$. Here, we find that $\left|\mathcal{T}_{y}\right| \approx 1 \%$ of the total torque $|\mathcal{T}|$. When the spin-orbit coupling $\gamma_{2}$ increases from 0 to 2.7, the spatial total out-of-plane torque $\left\langle\left|\mathcal{T}_{y}\right|\right\rangle$ increases by a factor of 1000. This explains the dramatic increase in the domain wall drift velocity and thereby mobility.
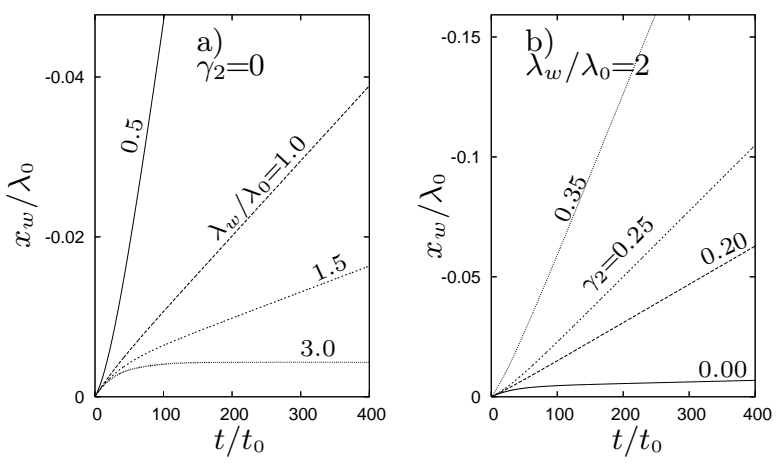

FIG. 3: Domain wall displacement as a function of time for a) zero spin-orbit coupling for different domain wall widths, and b) fixed domain wall width for varying hole spin-orbit coupling strengths. For both plots, $h_{p d}=0.001$.

Fig 3 a displays the domain wall displacement $\left(x_{w}\right)$ as functions of time for varying domain wall widths for vanishing spin-orbit coupling. $x_{w}$ completely saturates in the adiabatic limit, $\lambda_{w} / \lambda_{0}>3$, as expected [10, 11, 15]. Beyond the adiabatic limit, an increasing fraction of holes will be reflected, causing a out-of-plane torque on the domain wall and a finite drift velocity, which, as can be seen in Fig 3), increases for decreasing domain wall width.

Let us now consider a finite spin-orbit coupling. Fig (3b) shows the domain wall displacement as a function of time for different spin-orbit couplings. We see that a larger spin-orbit coupling also increases the domain wall drift velocity due to the increased reflection of holes. The reflected holes increase the spin accumulation and mistracking, which, in turn, increases the out-of-plane spintorque on the domain wall and thereby its drift velocity.

Fig (4a) shows the domain wall drift velocity as a function of $h_{p d}$ for varying spin-orbit coupling strength. We see that $v_{w}$ is proportional to $h_{p d}$, i.e. the domain wall drift velocity is proportional to the current density, consistent with experimental observations [1]. In Fig 4b), we show the current-driven domain wall mobility as a function of spin-orbit coupling. We see that the domain wall mobility increases four orders of magnitude when the hole spin-orbit coupling increases from $\gamma_{2}=0$ to a typical value 

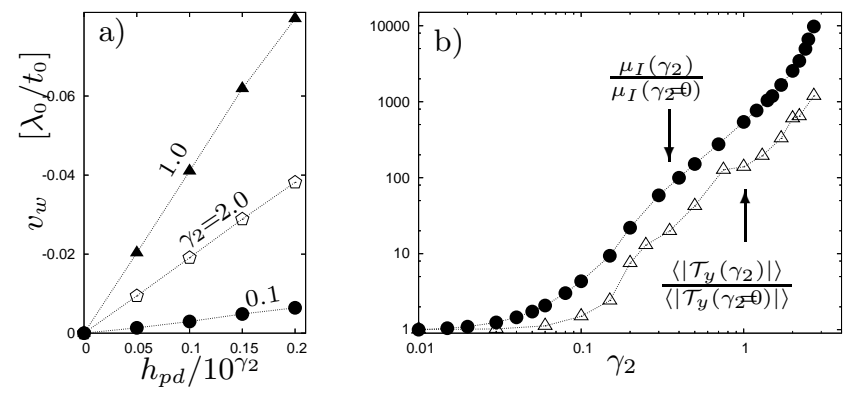

FIG. 4: a) Domain wall drift velocity as a function of $h_{p d}$ for varying spin-orbit couplings, $\gamma_{2}$. Note that the scale of the horizontal axis is different for each spin-orbit coupling, done to collect all graphs into a single plot. b) Current-driven domain wall mobility, $\mu_{I}$, and spatial total of the absolute values of the out-of-plane torque, $\left\langle\left|\mathcal{T}_{y}\right|\right\rangle$, as functions of spinorbit coupling. For both plots, $\lambda_{w} / \lambda_{0}=2$. Lines are guides for the eye.

of $\gamma_{2}=2.7$. In Fig 4 b), we also show the spatial total outof-plane torque as a function of the spin-orbit coupling. We see that $\left\langle\left|\mathcal{T}_{y}\left(\gamma_{2}\right)\right|\right\rangle /\left\langle\left|\mathcal{T}_{y}\left(\gamma_{2}=0\right)\right|\right\rangle$ increases three orders of magnitude when $\gamma_{2}$ increases from 0 to 2.7. Increasing spin-orbit coupling increases the out-of-plane torque and thereby the mobility.

The effects of impurities [26] have been disregarded here. However, we believe that the present study still captures the essential physics. For example, we find that the intrinsic domain wall resistance persists in the diffusive transport regime [27]. Therefore, we expect that the spin-orbit induced effects presented in this paper are important also for the current-driven domain wall dynamics in the diffusive transport regime when the mean free path is not much smaller than $\lambda_{w}$. The expected impurity induced reduction of the domain wall drift velocity is consistent with recent experimental findings [1, 2, 3]. Experiments find a two to three orders of magnitude enhancement of the drift velocity, which is smaller than our computed three to four order of magnitude enhancement in the ballistic regime.

In summary, the intrinsic spin-orbit coupling in (Ga,Mn)As increases the out-of-plane torque on the domain wall and thereby the domain wall current-mobility by three to four orders of magnitude when the spin-orbit coupling increases from $\gamma_{2}=0$ to an experimentally attainable value of $\gamma_{2}=2.7$.

We thank G. E. W. Bauer, J. Hove, K. Olaussen, G. Tatara, and Y. Tserkovnyak for stimulating discussions.
This work has been supported by the Research Council of Norway through grants no. 167498/V30, 162742/V00, $1585181 / 143$, and $1585471 / 431$.

[1] M. Yamanouchi, D. Chiba, F. Matsukura, T. Dietl, H. Ohno, Phys. Rev. Lett. 96, 96601 (2006).

[2] M. Yamanouchi, D. Chiba, F. Matsukura and H. Ohno, Nature 428, 539 (2004).

[3] C. Gould et al., Japanese J. App. Phys. 45, 3860 (2006).

[4] A. Yamaguchi et al., Phys. Rev. Lett. 92, 077205 (2004).

[5] D. Chiba et al., Phys. Rev. Lett. 96, 096602 (2006).

[6] P. P. Freitas and L. Berger, J. App. Phys. 57, 1266 (1985); L. Gan et al., IEEE Trans. Magn. 36, 3047 (2000); J. Grollier et al., App. Phys. Lett. 83, 509 (2003); E. Saitoh, H. Miyajima, T. Yamaoka, and G. Tatara, Nature 432, 203 (2004).

[7] J. C. Slonczewski, J. Magn. Magn. Mat. 159, L1 (1996).

[8] L. Berger, Phys. Rev. B 33, 1572 (1986).

[9] Y. B. Bazaliy, B. A. Jones, and S.-C. Zhang, Phys. Rev. B 57, R3213 (1998).

[10] Z. Li and S. Zhang, Phys. Rev. Lett. 92 , 207203 (2004).

[11] G. Tatara and H. Kohno, Phys. Rev. Lett. 92, 086601 (2004).

[12] S. Zhang and Z. Li, Phys. Rev. Lett. 93, 127204 (2004).

[13] A. Thiaville et al., Europhys. Lett. 69, 990 (2005); Y. Tserkovnyak, H. J. Skadsem, A. Brataas, and G. E. W. Bauer, Phys. Rev. B 74, 144405 (2006); H. Kohno, G. Tatara and J. Shibata, J. Phys. Soc. Japan 75, 113706 (2006).

[14] J. Xiao, A. Zangwill and M. D. Stiles, Phys. Rev. B 73, 054428 (2006).

[15] J.I.Ohe and B. Kramer, Phys. Rev. Lett. 96,27204 (2006)

[16] C. H. Marrows, Adv. Phys. 54, 585 (2005).

[17] A. S. Núñez and A. H. MacDonald, cond-mat/0403710.

[18] A. K. Nguyen, R. V. Shchelushkin and A. Brataas, Phys. Rev. Lett. 97, 136603 (2006).

[19] R. Oszwaldowski, J. A. Majewski and T. Dietl, Phys. Rev. B 74, 153310 (2006).

[20] J. M. Luttinger, Phys. Rev. 102, 1030 (1956); A. Baldereschi and N. O. Lipari, Phys. Rev. B 8, 2697 (1973).

[21] J. Schliemann, Phys. Rev. B 74, 045214 (2006).

[22] T. Jungwirth, J. Sinova, J. Mašek, J. Kučera, and A. H. MacDonald, Rev. Mod. Phys. 78, 809 (2006).

[23] T. Usuki, M. Saito, M. Takatsu, R. A. Kiehl, N. Yokoyama, Phys. Rev. B 52, 8244 (1995).

[24] J. Z. Sun, Phys. Rev. B 62, 570 (2000).

[25] J. Sinova et al., Phys. Rev. B 69, 085209 (2004); Y. Tserkovnyak, G. A. Fiete, and B. I. Halperin, Appl. Phys. Lett. 84, 5234 (2004).

[26] C. Timm, J. Phys.: Condens. Matter 15 , R1865 (2003).

[27] A. K. Nguyen, R. V. Shchelushkin and A. Brataas, in preparation. 